\title{
Increased Visit-to-Visit Liver Enzyme Variability Is Associated with Incident Diabetes: A Community- Based 12-Year Prospective Cohort Study
}

\author{
Kyuhoon Bang, Ji Eun Jun, In-Kyung Jeong, Kyu Jeung Ahn, Ho Yeon Chung, You-Cheol Hwang \\ Division of Endocrinology and Metabolism, Department of Medicine, Kyung Hee University Hospital at Gangdong, Kyung Hee University School of Medicine, \\ Seoul, Korea
}

Background: Fatty liver and/or increased liver enzyme values have been reported to be associated with incident diabetes. We sought to determine whether increased visit-to-visit liver enzyme variability is associated with incident diabetes.

Methods: Study participants were recruited from the Korean Genome and Epidemiologic Study (KoGES). A total of 4,151 people aged 40 to 69 years was recruited and tested every 2 years for up to 12 years. Visit-to-visit aspartate aminotransferase (AST) and alanine aminotransferase (ALT) variability was evaluated in first the 6-year period through the use of various variability measurements: standard deviation (SD), average successive variability, coefficient of variation (CV), and variation independent of mean (VIM). Oral glucose tolerance test was performed at every visit.

Results: During the 6-year follow-up appointments, 13.0\% (538/4,151) of people developed incident diabetes. Visit-to-visit AST variability was associated with an increased risk of diabetes independent of conventional risk factors for diabetes (hazard ratio per 1-SD increment [95\% confidence interval]: 1.06 [1.00 to 1.11], 1.12 [1.04 to 1.21], and 1.13 [1.04 to 1.22] for SD, CV, and VIM, respectively; all $P<0.05$ ); however, no such associations were observed in the visit-to-visit ALT variability. According to alcohol consumption status, both AST and ALT variability were independent predictors for incident diabetes in subjects with heavy alcohol consumption; however, neither AST nor ALT variability was associated with diabetes risk in subjects who did not drink alcohol heavily.

Conclusion: Visit-to-visit liver enzyme variability is an independent predictor of incident diabetes. Such association was more evident in those who consumed significant amounts of alcohol.

Keywords: Alanine transaminase; Aspartate aminotransferases; Biological variation; Cohort studies; Diabetes mellitus, type 2; Korea

\section{INTRODUCTION}

Accumulating epidemiologic evidence shows that non-alcoholic fatty liver disease (NAFLD) and type 2 diabetes mellitus (T2DM) commonly coexist, and up to $70 \%$ to $80 \%$ of patients with T2DM are reported to be affected by NAFLD [1]. These two disease entities have common conditions and pathophysiologic mechanisms including obesity, insulin resistance, and pro- inflammatory and proatherogenic states and mutually increase the risk of developing more severe forms of NAFLD and cardiovascular complications, respectively [1,2]. Furthermore, growing evidence suggests that NAFLD often precedes development of T2DM [3]. In a meta-analysis performed with 19 observational studies with almost 300,000 individuals $(30.1 \%$ with NAFLD), patients with NAFLD showed a 2.2-fold increased risk in incident diabetes than those without NAFLD [4].
Corresponding author: You-Cheol Hwang (D) https://orcid.org/0000-0003-4033-7874 Division of Endocrinology and Metabolism, Department of Medicine, Kyung Hee University Hospital at Gangdong, Kyung Hee University School of Medicine,

892 Dongnam-ro, Gangdong-gu, Seoul 05278, Korea

E-mail:khmcilyong@naver.com
This is an Open Access article distributed under the terms of the Creative Commons Attribution Non-Commercial License (https://creativecommons.org/licenses/by-nc/4.0/) which permits unrestricted non-commercial use, distribution, and reproduction in any medium, provided the original work is properly cited. 
Recent studies have suggested that increased visit-to-visit variability in diverse clinical and biochemical measurements is independently associated with cardiovascular events [5-8]. Among patients with T2DM, body weight variability was associated with higher rates of cardiovascular events and mortality independent of traditional cardiovascular risk factors [5]. In addition, in a meta-analysis from prospective cohort studies or clinical trials in adults, increased long term variability in systolic blood pressure was associated with the risk of cardiovascular events and all-cause and cardiovascular mortality [7]. Similarly, in an analysis of the TNT trial data, visit-to-visit lowdensity lipoprotein cholesterol (LDL-C) variability was associated with future cardiovascular events independent of treatment effect and achieved mean LDL-C levels [8].

Based on these observations, we sought to determine whether increased visit-to-visit liver enzyme variability is an independent predictor of incident diabetes using data from a community-based prospective cohort in Korean adults. In addition, we further clarified the association between visit-to-visit liver enzyme variability and diabetes risk differs according to alcohol consumption status.

\section{METHODS}

\section{Study population}

The Korean Genome and Epidemiologic Study (KoGES) is a prospective study that was launched by the Korean Center for Disease Control and Prevention. In this study, participants were recruited from two communities in South Korea: the Ansung cohort for a rural community and the Ansan cohort for an urban community (the Ansung-Ansan Cohort Study). Baseline examinations were performed in 2001 to 2002; the follow-up examinations were ongoing every 2 years, and data up to 2013 to 2014 were used in this study. Details regarding the study's design and baseline characteristics and follow-up rate at every visit have been published in previous study [9]. During the 12-year study period, we used data from the first 6 years, which included a total of four clinic visits, to calculate liver enzyme variability ('baseline period,' from 2001-2002 to 2007-2008), and the data from the remaining 6 years were used to determine incident diabetes ('follow-up period', from 2007-2008 to 2013-2014). Thus, year 2007 to 2008 was designated as the new baseline in this study. A total of 10,030 subjects aged 40 to 69 years was recruited, among whom 2,397 subjects with diabetes based on self-reported history, use of antidiabetic drugs and/or insulin, and oral glucose tolerance test (OGTT) during 'baseline period' and/or 423 subjects with a history of hepatitis at baseline examination (2001 to 2002) were excluded. Among the remaining 7,326 subjects, we limited study subjects to 4,396 who were followed up every visit during the 'baseline period' and then further excluded subjects with missing aspartate aminotransferase (AST) and alanine aminotransferase (ALT) levels $(n=40)$ and/or body weight measurements during the 'baseline period' $(n=51)$. Among the remaining 4,331 subjects, 4,151 who had follow-up OGTT data collected at least once during the 'follow-up period' were enrolled in this study (Supplementary Fig. 1).

Informed consent was obtained from all study subjects. The study protocol was approved by the Ethics Committee of the Korean Center for Disease Control and the Kyung Hee University Hospital at Gangdong Institutional Review Board (KHNMC IRB 2020-02-003).

\section{Clinical and biochemical parameters}

Body mass index (BMI) was calculated as weight in kilograms divided by the square of height in meters $\left(\mathrm{kg} / \mathrm{m}^{2}\right)$ in light clothing. Waist circumference was measured at the midpoint between the lower limit of the ribcage and the iliac crest. Smoking status was classified into three groups: current smoker, past smoker, and never smoker. Heavy alcohol drinker was defined as alcohol consumption $\geq 30$ g/day for men and $\geq 20$ $\mathrm{g} /$ day for women [10]. Regular physical activity was defined as moderate or vigorous intensity physical activity that lasted $\geq 30$ minutes daily.

After overnight fasting for 12 hours, blood samples were collected and analyzed at a central laboratory (Seoul Clinical Laboratories, Seoul, Korea). The plasma concentrations of glucose, total cholesterol, triglycerides, and high-density lipoprotein cholesterol (HDL-C) were measured enzymatically using a 747 Chemistry Analyzer (Hitachi, Tokyo, Japan). White blood cell (WBC) counts were measured using an autoanalyzer (Sysmex, Kobe, Japan). Levels of blood urea nitrogen and creatinine, as well as ALT and AST, were measured using a Hitachi 747 automated analyzer. High-sensitivity C-reactive protein (hsCRP) concentrations were measured by immunoradiometric assay (ADVIA 1650; Bayer Diagnostics, Tarrytown, NY, USA). Glycosylated hemoglobin (HbA1c) levels were measured by highperformance liquid chromatography (VARIANT II; Bio-Rad Laboratories, Hercules, CA, USA); however, HbAlc was not used to define glucose tolerance categories since it was not in- 
corporated in the diagnostic criteria at the start of the study. Plasma insulin concentrations were determined using a radioimmunoassay kit (Linco Research, St. Charles, MO, USA).

\section{Definitions}

All participants underwent a $75 \mathrm{~g}$ OGTT at inclusion and then every 2 years. Plasma glucose and insulin concentrations were measured at 0,60, and 120 minutes during the OGTT. The definitions of diabetes were based on plasma glucose levels during the $75 \mathrm{~g}$ OGTT, as defined by the 1997 American Diabetes Association criteria [11]. To determine the subject's insulin secretory capacity, a homeostasis model assessment of $\beta$ cell function (HOMA-B\%) was calculated as $20 \times$ [fasting insulin $(\mu \mathrm{U} / \mathrm{mL})] \times[$ fasting glucose-3.5 $(\mathrm{mmol} / \mathrm{L})]$ [12]. In addition, the insulinogenic index (IGI), which is an estimate of early phase insulin secretion, was calculated by dividing the incremental change in insulin during the first 60 -minute by the corresponding change in glucose $[60-0$ minutes insulin $(\mathrm{IU} / \mathrm{mL}) /$ 60-0 minutes glucose $(\mathrm{mg} / \mathrm{mL})]$ [13]. Insulin sensitivity was estimated by homeostasis model assessment of insulin resistance (HOMA-IR), which was calculated as [fasting glucose $(\mathrm{mg} / \mathrm{dL}) \times$ fasting insulin $(\mu \mathrm{U} / \mathrm{mL})] / 405$ [12]. In addition, the composite (Matsuda) insulin sensitivity index (ISI) was calculated as $10,000 /[$ fasting glucose $(\mathrm{mg} / \mathrm{dL}) \times$ fasting insulin $(\mu \mathrm{U} /$ $\mathrm{mL}) \times$ mean glucose $(\mathrm{mg} / \mathrm{dL}) \times$ mean insulin $(\mu \mathrm{U} / \mathrm{mL})]^{0.5}$ using 0,60 , and 120 minutes values from the OGTT [14]. To determine pancreatic $\beta$-cell function adjusting for insulin sensitivity, the oral disposition index was calculated by multiplying the IGI $_{60}$ by the composite ISI [13].

Hypertension was defined as one of the following: (1) selfreported previous history of hypertension; (2) systolic or diastolic blood pressure $\geq 140 / 90 \mathrm{~mm} \mathrm{Hg}$; or (3) use of antihypertensive medications [15]. Hyperlipidemia was defined as one of the following: (1) self-reported previous history of lipid abnormalities; (2) total cholesterol $\geq 240 \mathrm{mg} / \mathrm{dL}$; or (3) use of lipid-lowering medications [16]. A cardiovascular disease (CVD) event was defined as either myocardial infarction, coronary artery disease, congestive heart failure, stroke, or peripheral artery disease, and data on each event were obtained from the study participants' reports. The CVD events reported by participants were corroborated by an in-depth interview during the initial examination and at interviews repeated at each biennial follow-up visit. To calculate visit-to-visit AST and ALT variability, various measurements were used as follows: (1) the standard deviation (SD) of AST and ALT levels; (2) the average successive variability, which was defined as the average absolute difference between successive values; (3) coefficient of variation (CV); and (4) variation independent of mean (VIM). VIM was calculated as $100 \times \mathrm{SD} /$ mean $^{\text {beta }}$, where beta is the regression coefficient, on the basis of natural logarithm of SD on natural logarithm of mean [8].

\section{Statistical analysis}

Data are expressed as mean $\pm \mathrm{SD}$ for continuous measures or as proportion for categorical variables. Differences between groups were tested by Student's $t$-test for continuous variables and the chi-square test for categorical variables. Spearman's correlation coefficients were performed to determine the associations between liver enzyme variability and indices reflecting insulin secretion, insulin resistance, and low-grade inflammation. Hazard ratios (HRs) with 95\% confidence intervals (CIs) were calculated for independent variables included in a Cox proportional hazards model, with a 1-SD increment used for HR calculations for continuous measurements. A variance inflation factor $>5.0$ was used as an indicator of multicollinearity. The presence of interaction was assessed in multivariate models by testing the significance of first-order interaction terms. Analyses were performed using R version 3.6.2 (http:// www.r-project.org) and SPSS software version 24.0 (IBM Co., Armonk, NY, USA). $P$ values $<0.05$ were considered statistically significant.

\section{RESULTS}

Of 4,151 participants free of diabetes during the 'baseline period,' 538 individuals (13.0\%) developed diabetes during the 6-year 'follow-up period.' Individuals who developed diabetes were older, more obese, and showed unfavorable metabolic phenotypes, including higher blood pressure and glucose levels, atherogenic dyslipidemic profiles, and insulin resistance compared with individuals who did not develop diabetes. In addition, individuals with incident diabetes showed elevated liver enzyme levels, including AST and ALT, and inflammatory markers, which were determined by WBC counts and hsCRP levels, compared with individuals who did not develop diabetes. However, daily alcohol consumption amount and the proportion of heavy alcohol drinkers were not different between the two groups (Table 1).

Similarly, the univariate Cox hazards model showed age, BMI, waist circumference, and other traditional risk factors for 
Table 1. Baseline (year 2007 to 2008) characteristics

\begin{tabular}{|c|c|c|c|c|}
\hline Characteristic & $\begin{array}{c}\text { Total } \\
(n=4,151)\end{array}$ & $\begin{array}{l}\text { Incident diabetes }(-) \\
\qquad(n=3,613)\end{array}$ & $\begin{array}{l}\text { Incident diabetes }(+) \\
\qquad(n=538)\end{array}$ & $P$ value \\
\hline Age, yr & $57.1 \pm 8.5$ & $56.9 \pm 8.5$ & $58.6 \pm 8.6$ & $<0.001$ \\
\hline Male sex & $1,874(45.1)$ & $1,638(45.3)$ & $236(43.9)$ & 0.553 \\
\hline Body mass index, $\mathrm{kg} / \mathrm{m}^{2}$ & $24.3 \pm 3.0$ & $24.2 \pm 2.9$ & $25.0 \pm 3.3$ & $<0.001$ \\
\hline Waist circumference, $\mathrm{cm}$ & $83.5 \pm 9.5$ & $83.2 \pm 9.4$ & $85.6 \pm 10.2$ & $<0.001$ \\
\hline Current smoker & $1,414(34.1)$ & $1,229(34.1)$ & $185(34.4)$ & 0.921 \\
\hline Daily alcohol consumption, g/day & $18.4 \pm 25.5$ & $18.1 \pm 25.0$ & $20.0 \pm 28.6$ & 0.327 \\
\hline Heavy alcohol drinker & $388(9.4)$ & $328(9.1)$ & $60(11.2)$ & 0.143 \\
\hline Regular physical activity & $696(16.8)$ & $609(16.9)$ & $87(16.2)$ & 0.738 \\
\hline Family history of diabetes & $426(10.3)$ & $361(10.0)$ & $65(12.1)$ & 0.157 \\
\hline Hypertension & $1,187(28.7)$ & $986(27.4)$ & $201(37.4)$ & $<0.001$ \\
\hline Hyperlipidemia & $502(12.1)$ & $417(11.6)$ & $85(15.8)$ & 0.006 \\
\hline Cardiovascular disease & $64(1.5)$ & $51(1.4)$ & $13(2.4)$ & 0.116 \\
\hline Systolic blood pressure, $\mathrm{mm} \mathrm{Hg}$ & $116.2 \pm 15.7$ & $115.8 \pm 15.5$ & $119.4 \pm 16.2$ & $<0.001$ \\
\hline Diastolic blood pressure, $\mathrm{mm} \mathrm{Hg}$ & $75.0 \pm 9.2$ & $74.8 \pm 9.2$ & $76.2 \pm 9.2$ & 0.001 \\
\hline Fasting plasma glucose, $\mathrm{mg} / \mathrm{dL}$ & $91.2 \pm 8.7$ & $90.5 \pm 8.2$ & $96.0 \pm 10.5$ & $<0.001$ \\
\hline 2-Hour post-load glucose, $\mathrm{mg} / \mathrm{dL}$ & $121.9 \pm 31.1$ & $119.5 \pm 29.7$ & $138.8 \pm 34.6$ & $<0.001$ \\
\hline Fasting serum insulin, $\mu \mathrm{IU} / \mathrm{mL}$ & $8.56 \pm 4.54$ & $8.45 \pm 4.56$ & $9.33 \pm 4.37$ & $<0.001$ \\
\hline HOMA-IR & $1.95 \pm 1.14$ & $1.91 \pm 1.14$ & $2.23 \pm 1.13$ & $<0.001$ \\
\hline Total cholesterol, mg/dL & $196.0 \pm 34.0$ & $195.9 \pm 33.7$ & $196.6 \pm 36.3$ & 0.667 \\
\hline HDL-C, mg/dL & $44.8 \pm 10.6$ & $45.1 \pm 10.6$ & $42.9 \pm 10.3$ & $<0.001$ \\
\hline Triglycerides, mg/dL & $132.1 \pm 81.8$ & $128.8 \pm 77.9$ & $154.2 \pm 101.7$ & $<0.001$ \\
\hline Blood urea nitrogen, $\mathrm{mg} / \mathrm{dL}$ & $15.9 \pm 4.4$ & $15.9 \pm 4.4$ & $15.7 \pm 4.2$ & 0.456 \\
\hline Creatinine, $\mathrm{mg} / \mathrm{dL}$ & $0.93 \pm 0.21$ & $0.93 \pm 0.22$ & $0.94 \pm 0.17$ & 0.801 \\
\hline Aspartate aminotransferase, $\mathrm{U} / \mathrm{L}$ & $25.7 \pm 11.5$ & $25.5 \pm 10.3$ & $27.2 \pm 17.6$ & 0.034 \\
\hline Alanine aminotransferase, U/L & $23.5 \pm 14.9$ & $23.2 \pm 14.7$ & $25.1 \pm 16.1$ & 0.013 \\
\hline White blood cell, number/mL & $6,017 \pm 1,368$ & $5,980 \pm 1,351$ & $6,261 \pm 1,451$ & $<0.001$ \\
\hline High sensitivity C-reactive protein, mg/L & $1.08 \pm 1.35$ & $1.03 \pm 1.30$ & $1.40 \pm 1.58$ & $<0.001$ \\
\hline
\end{tabular}

Values are presented as mean \pm standard deviation or number (\%). To convert glucose level to millimoles per liter, multiply by 0.0555 . To convert insulin level to picomoles per liter, multiply by 6.945. To convert total, low-density lipoprotein cholesterol, and HDL-C levels to millimoles per liter, multiply by 0.0259 . To convert triglyceride level to millimoles per liter, multiply by 0.0113 .

HOMA-IR, homeostasis model assessment of insulin resistance; HDL-C, high-density lipoprotein cholesterol.

diabetes were associated with development of diabetes. Again, however, daily alcohol consumption amount or heavy alcohol drinking was not associated with incident diabetes. Regarding variables from AST and ALT measurements, mean AST and ALT values during 'baseline period' were associated with diabetes risk. For every 1-SD increase, the risk of diabetes increased by $5 \%$ (HR per $1-\mathrm{SD}, 1.05$; $95 \% \mathrm{CI}, 1.00$ to 1.10 ; $P=$ 0.036). Similar associations were observed in CV (HR per 1-SD, 1.12; 95\% CI, 1.05 to 1.20; $P=0.001$ ) and VIM (HR per
1-SD, 1.10; 95\% CI, 1.01 to $1.19 ; P=0.020$ ) of AST and risk of diabetes. However, such associations were not observed between visit-to-visit ALT variability and incident diabetes (Table 2). In addition, the number of incident cases of diabetes increased according to AST and ALT variability tertile (Supplementary Fig. 2).

To determine whether visit-to-visit AST and ALT variability independently predicts future development of diabetes, a Cox proportional hazard regression model was fit that included 
Table 2. Univariate analysis for diabetes risk

\begin{tabular}{|c|c|c|}
\hline Variable & HR (95\% CI) & $P$ value \\
\hline Age & $1.22(1.12-1.32)$ & $<0.001$ \\
\hline Male sex & $0.98(0.83-1.17)$ & 0.843 \\
\hline Body mass index & $1.27(1.17-1.38)$ & $<0.001$ \\
\hline Waist circumference & $1.28(1.17-1.39)$ & $<0.001$ \\
\hline Current smoker & $1.06(0.89-1.27)$ & 0.520 \\
\hline Daily alcohol consumption & $1.07(0.96-1.20)$ & 0.230 \\
\hline Heavy alcohol drink & $1.27(0.97-1.66)$ & 0.079 \\
\hline Regular physical activity & $0.98(0.78-1.23)$ & 0.860 \\
\hline Family history of diabetes & $1.16(0.90-1.51)$ & 0.253 \\
\hline Hypertension & $1.58(1.32-1.88)$ & $<0.001$ \\
\hline Hyperlipidemia & $1.35(1.07-1.70)$ & 0.012 \\
\hline Cardiovascular disease & $1.65(0.95-2.86)$ & 0.076 \\
\hline Systolic blood pressure & $1.24(1.15-1.34)$ & $<0.001$ \\
\hline Diastolic blood pressure & $1.16(1.07-1.26)$ & $<0.001$ \\
\hline Fasting plasma glucose & $1.72(1.60-1.86)$ & $<0.001$ \\
\hline 2-Hour post-load glucose & $1.82(1.67-1.99)$ & $<0.001$ \\
\hline Fasting serum insulin & $1.11(1.06-1.17)$ & $<0.001$ \\
\hline HOMA-IR & $1.12(1.08-1.17)$ & $<0.001$ \\
\hline Total cholesterol & $1.01(0.93-1.10)$ & 0.763 \\
\hline HDL-C & $0.81(0.73-0.89)$ & $<0.001$ \\
\hline Triglycerides & $1.25(1.17-1.32)$ & $<0.001$ \\
\hline Blood urea nitrogen & $0.96(0.88-1.05)$ & 0.354 \\
\hline Creatinine & $1.01(0.95-1.07)$ & 0.815 \\
\hline White blood cell count & $1.18(1.09-1.29)$ & $<0.001$ \\
\hline High sensitivity C-reactive protein & $1.21(1.13-1.29)$ & $<0.001$ \\
\hline \multicolumn{3}{|l|}{ Aspartate aminotransferase } \\
\hline Baseline & $1.14(1.08-1.20)$ & $<0.001$ \\
\hline Mean & $1.10(1.04-1.16)$ & 0.001 \\
\hline $\mathrm{SD}$ & $1.05(1.00-1.10)$ & 0.036 \\
\hline $\mathrm{CV}$ & $1.12(1.05-1.20)$ & 0.001 \\
\hline ASV & $1.04(0.99-1.09)$ & 0.089 \\
\hline VIM & $1.10(1.01-1.19)$ & 0.023 \\
\hline \multicolumn{3}{|l|}{ Alanine aminotransferase } \\
\hline Baseline & $1.11(1.04-1.18)$ & 0.001 \\
\hline Mean & $1.09(1.04-1.14)$ & $<0.001$ \\
\hline $\mathrm{SD}$ & $1.03(0.97-1.09)$ & 0.419 \\
\hline $\mathrm{CV}$ & $1.03(0.95-1.11)$ & 0.533 \\
\hline ASV & $1.03(0.97-1.08)$ & 0.351 \\
\hline VIM & $0.97(0.89-1.06)$ & 0.541 \\
\hline
\end{tabular}

HR for continuous variables are shown as 1-SD increment. HR, hazard ratio; CI, confidence interval; HOMA-IR, homeostasis model assessment of insulin resistance; HDL-C, high-density lipoprotein cholesterol; SD, standard deviation; CV, coefficient of variation; ASV, average successive variability; VIM, variability independent of the mean. variables found to be significantly associated with diabetes risk in univariate analysis (Table 2). Since systolic and diastolic blood pressures are highly correlated and provide similar information, the former was chosen for inclusion in the multivariate model due to its higher HR in univariate analysis. Similarly, inclusion of both HOMA-IR and fasting insulin in a model causes multicollinearity; thus, we selected fasting insulin because HOMA-IR includes fasting glucose, another variable included in our multivariate model. In the fully adjusted model III, SD (HR per 1-SD, 1.06; 95\% CI, 1.00 to $1.11 ; P<$ 0.05), CV (HR per 1-SD, 1.12; 95\% CI, 1.04 to $1.21 ; P<0.01$ ), and VIM (HR per 1-SD, 1.13; 95\% CI, 1.04 to $1.22 ; P<0.01$ ) of AST were independently associated with future diabetes risk. On the other hand, visit-to-visit ALT variability was not associated with diabetes risk in the multivariate model. When we divided study participants according to alcohol intake status, AST and ALT variability were independently associated with diabetes risk in subjects who were heavy alcohol drinkers (model III); however, neither AST nor ALT variability was associated with diabetes risk in subjects who did not drink alcohol heavily except VIM of AST. In addition, these associations between liver enzyme variability and diabetes risk, particularly AST value in subjects who were heavy alcohol drinkers, was still evident even after further adjustment for mean AST or ALT levels, respectively (model IV) (Table 3).

Finally, we sought to determine which mechanisms are involved in the association between increased liver enzyme variability and diabetes risk. Spearman's correlation analysis showed that liver enzyme variability during the 'baseline period' appeared to be more closely related to insulin resistance determined by HOMA-IR and ISI compared with low insulin secretory capacity determined by HOMA-B\%, IGI, and the disposition index at baseline (year 2007 to 2008). In addition, increased low grade inflammation might be involved in the association between liver enzyme variability and diabetes risk (Table 4). Furthermore, we selected indices that reflected insulin secretion, insulin resistance, and low-grade inflammation based on simple linear regression analysis. Next, we put the selected indices into a multiple regression analysis model and compared standardized $\beta$ coefficients. As a result, insulin resistance appeared to be the highest associating factor with liver enzyme variability rather than insulin secretion defect or lowgrade inflammation (Supplementary Table 1). 

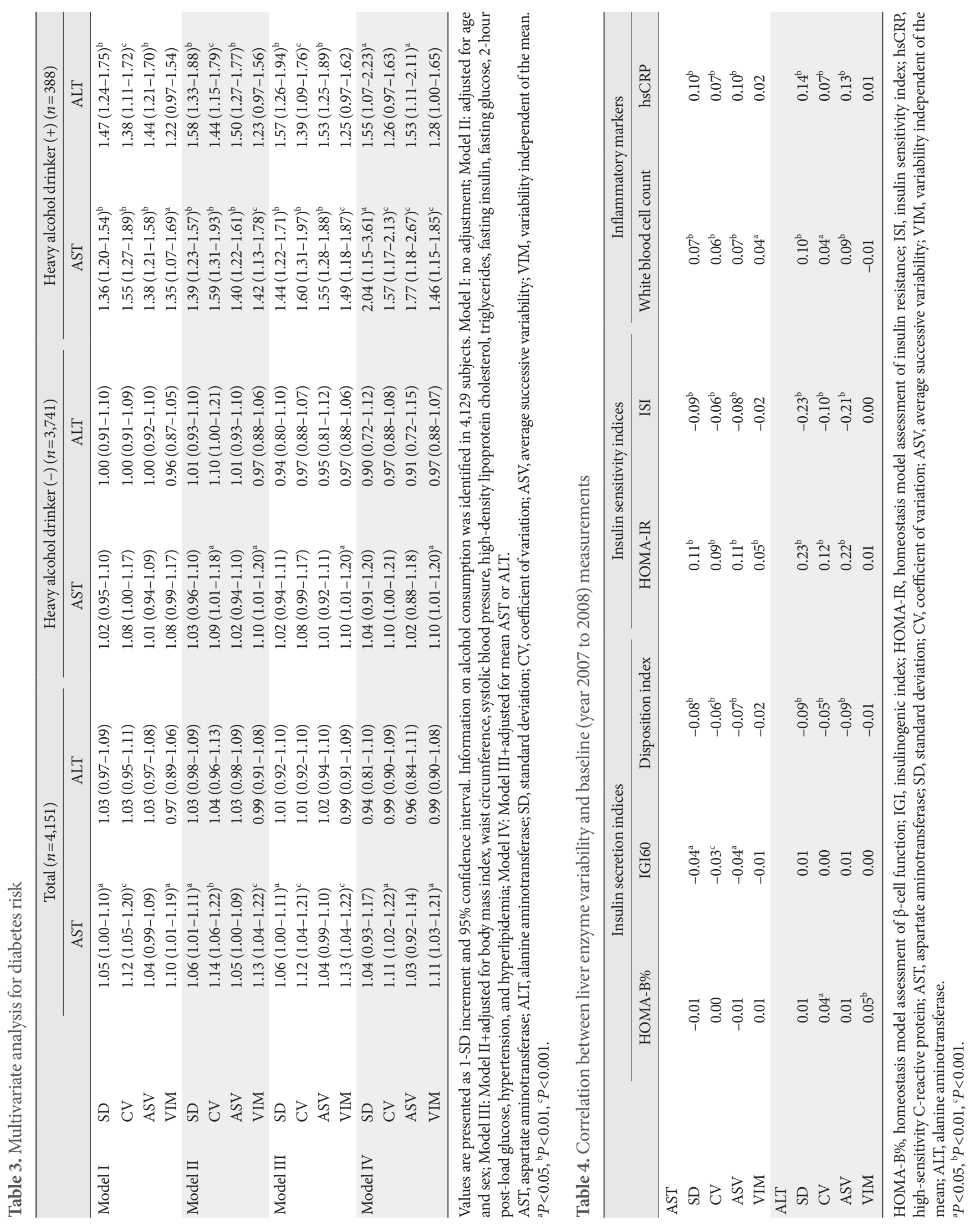


\section{DISCUSSION}

In this community-based prospective cohort study, visit-to-visit variability in liver enzyme AST and ALT levels were predictive of incident diabetes independent of traditional risk factors of diabetes and mean AST and ALT levels during the 6-year follow-up period. Additional findings from this study were (1) the predictive role of liver enzyme variability for incident diabetes was more evident in people with heavy alcohol consumption compared with casual or non-drinkers; (2) AST variability was more closely related to incident diabetes than was ALT variability; and (3) insulin resistance was more closely related to liver enzyme variability than was lower insulin secretion, and thus it could mediate diabetes risk with liver enzyme variability.

In addition to severity and duration of exposure in cardiovascular risk factors, recent data have suggested that visit-tovisit variability in cardiovascular risk factors including blood pressure, LDL-C, glucose, and body weight are associated with development of CVD independent of absolute values [17]. However, less is known about the associations between variability in cardiovascular risk factors and other metabolic outcomes such as diabetes risk. A recent study has shown that fasting glucose variability was predictive of incident diabetes even after adjusting for lifestyle parameters and anthropometric and laboratory variables including mean fasting glucose level during a follow-up period of 8.3 years [18]. In addition, a meta-analysis of eight studies indicates that people with the most unstable body weight had 33\% higher risk for developing diabetes compared with people with the most stable body weight [19]. In terms of lipid measurements, both higher total and HDL-C variability was associated with diabetes risk independent of the use of lipid-lowering medication [20,21]. Collectively, based on aforementioned studies, visit-to-visit variability in diverse cardiovascular risk factors also appears to be associated with incident diabetes. Thus, we sought to extend our knowledge about the association between visit-to-visit variability in traditional cardiovascular risk factors and outcomes to variability in other parameters that can be easily measured in routine clinical practice-in the current study, that was liver enzyme levels. In a recent nationwide population-based study in Korea, higher variability in AST, ALT, and $\gamma$-glutamyltransferase levels was associated with a higher risk for all-cause mortality, myocardial infarction, atrial fibrillation, and congestive heart failure. In addition, these findings were consistent regardless of baseline characteristics including alco- hol consumption, BMI, and degree of fatty liver [22]. However, to our best knowledge, our current study is the first report to determine the association between visit-to-visit liver enzyme variability and diabetes risk independent of traditional risk factors of diabetes and mean levels.

In this study, the association between visit-to-visit liver enzyme variability and diabetes risk was more evident in people with heavy alcohol consumption compared with casual or non-drinkers and in AST variability rather than in ALT variability. However, daily alcohol consumption amount and heavy alcohol consumption per se were not associated with diabetes risk in our univariate model (Table 2). In addition, further adjustment of alcohol consumption variability or body weight variability to our fully adjusted model did not affect the association between liver enzyme variability and diabetes risk (data not shown). Therefore, changes in alcohol intake or body weight alone appeared to not entirely affect fluctuation in liver enzymes and the resulting increased diabetes risk. Instead, heavy alcohol drinkers were more likely to be male smokers and more likely to have unfavorable metabolic features including higher waist circumference, blood pressure, fasting glucose, and hsCRP levels compared with casual or non-drinker (data not shown). Thus, although we cannot explain the reason at this time, increased visit-to-visit liver enzyme variability may play a predictive role particularly in heavy alcohol drinkers with a higher baseline risk for incident diabetes compared with casual or non-drinkers.

In a previous study performed with the same KoGES cohort, Cho et al. [23] reported that baseline ALT level was a significant predictor for incident diabetes; however, AST was not. These results were somewhat different from ours; however, there are several possible explanations for the discrepancy between baseline liver enzyme level and their variability for future diabetes risk. First, although these two studies used data from same cohort, the study by Cho et al. [23] used liver enzyme data from 2001 to 2002 as a baseline and determined incident diabetes after a 2-year follow-up period. However, in our study, we calculated liver enzyme variability using data from 2001-2002 to 2007-2008, and determined incident diabetes using data from 2007-2008 to 2013-2014. Thus, the design differences between the two studies may explain the discrepancy. Second, analyzing liver enzyme data taken at one time point (baseline) or across a period of time (variability) could produce different results with regard to diabetes risk.

There are ongoing debates as to whether increased variability 
in cardiovascular risk factors increases the risk for cardiovascular events or if it is merely a predictor of cardiovascular events [17]. At present, some uncertainty exists between liver enzyme variability and diabetes risk. In addition, we do not know the precise mechanism for the effect of liver enzyme variability on glucose metabolism; however, correlation analysis and multiple linear regression analysis indicated that liver enzyme variability was more closely related to insulin resistance and inflammation than to insulin secretion (Table 4 and Supplementary Table 1). Thus, changes in insulin sensitivity and/or low-grade metabolic inflammation might be important mediators of diabetes risk with liver enzyme variability than of changes in insulin secretory capacity.

Our study has several limitations. First, we did not have access to viral markers for hepatitis B and C, and we could not exclude the patients with viral hepatitis from our analysis. However, we excluded patients with a history of hepatitis ( $n=$ $423,4.2 \%$ ), and recent epidemiologic studies reported that the prevalence of hepatitis B and C in adults was $2.9 \%$ and $0.7 \%$ in Korea, respectively $[24,25]$. Thus, although we could not fully exclude patients with viral hepatitis from this study, a considerable number of patients could be excluded and the possible remaining patients in our analysis would likely not have a decisive effect on our conclusions. Second, other biochemical tests and imaging techniques including $\gamma$-glutamyltransferase and ultrasound to determine liver function and steatosis were not available for this study. Third, seasonal variation in liver function tests has been reported [26]; however, we did not incorporate seasonal variation into our analysis. Fourth, we did not have information regarding medications that could possibly affect liver enzyme levels. Lastly, this finding cannot be generalized to other ethnicities because only a Korean population was included. Despite these limitations, our findings are powered by use of one of the most validated Korean communitybased prospective cohort dataset with a relatively large sample size and high follow-up rate $(4,151 / 4,331,95.8 \%)$. In addition, we proposed for the first time a role of liver enzyme variability as a novel risk factor for incident diabetes.

In conclusion, visit-to-visit liver enzyme variability is an independent predictor of incident diabetes. In clinical practice, physicians need to pay attention to patients' liver enzyme levels not only to assess liver function, but also to assess diabetes risk with visit-to-visit variability. In addition, future interventional studies are necessary to determine whether stabilizing liver enzyme variability would be beneficial for preventing diabetes risk.

\section{SUPPLEMENTARY MATERIALS}

Supplementary materials related to this article can be found online at https://doi.org/10.4093/dmj.2020.0208.

\section{CONFLICTS OF INTEREST}

No potential conflict of interest relevant to this article was reported.

\section{AUTHOR CONTRIBUTIONS}

Conception or design: Y.C.H.

Acquisition, analysis, or interpretation of data: K.B., J.E.J., I. K.J., K.J.A., H.Y.C., Y.C.H.

Drafting the work or revising: K.B., Y.C.H.

Final approval of the manuscript: K.B., J.E.J., I.K.J., K.J.A., H.Y.C., Y.C.H.

\section{ORCID}

Kyuhoon Bang https://orcid.org/0000-0001-7073-7196

You-Cheol Hwang https://orcid.org/0000-0003-4033-7874

\section{FUNDING}

This research was supported by the Bio \& Medical Technology Development Program of the National Research Foundation (NRF) funded by the Korean government (MSIT) (No. 2018M3A9E807881113).

\section{ACKNOWLEDGMENTS}

Data in this study were from the Korean Genome and Epidemiology Study (KoGES; 4851-302), National Research Institute of Health, Centers for Disease Control and Prevention, Ministry for Health and Welfare, Republic of Korea.

The authors thank Soojin Kim from the Institute of Medical Science, Kyung Hee University Hospital at Gangdong for statistical assistance.

\section{REFERENCES}

1. Lee YH, Cho Y, Lee BW, Park CY, Lee DH, Cha BS, et al. Nonalcoholic fatty liver disease in diabetes. Part I: epidemiology 
and diagnosis. Diabetes Metab J 2019;43:31-45.

2. Targher G, Lonardo A, Byrne CD. Nonalcoholic fatty liver disease and chronic vascular complications of diabetes mellitus. Nat Rev Endocrinol 2018;14:99-114.

3. Valenti L, Bugianesi E, Pajvani U, Targher G. Nonalcoholic fatty liver disease: cause or consequence of type 2 diabetes? Liver Int 2016;36:1563-79.

4. Mantovani A, Byrne CD, Bonora E, Targher G. Nonalcoholic fatty liver disease and risk of incident type 2 diabetes: a metaanalysis. Diabetes Care 2018;41:372-82.

5. Bangalore S, Fayyad R, DeMicco DA, Colhoun HM, Waters DD. Body weight variability and cardiovascular outcomes in patients with type 2 diabetes mellitus. Circ Cardiovasc Qual Outcomes 2018;11:e004724.

6. Mehlum MH, Liestol K, Kjeldsen SE, Julius S, Hua TA, Rothwell PM, et al. Blood pressure variability and risk of cardiovascular events and death in patients with hypertension and different baseline risks. Eur Heart J 2018;39:2243-51.

7. Stevens SL, Wood S, Koshiaris C, Law K, Glasziou P, Stevens $\mathrm{RJ}$, et al. Blood pressure variability and cardiovascular disease: systematic review and meta-analysis. BMJ 2016;354:14098.

8. Bangalore S, Breazna A, DeMicco DA, Wun CC, Messerli FH; TNT Steering Committee and Investigators. Visit-to-visit lowdensity lipoprotein cholesterol variability and risk of cardiovascular outcomes: insights from the TNT trial. J Am Coll Cardiol 2015;65:1539-48.

9. Kim Y, Han BG; KoGES group. Cohort profile: the Korean Genome and Epidemiology Study (KoGES) consortium. Int J Epidemiol 2017;46:1350.

10. Sanyal AJ, Brunt EM, Kleiner DE, Kowdley KV, Chalasani N, Lavine JE, et al. Endpoints and clinical trial design for nonalcoholic steatohepatitis. Hepatology 2011;54:344-53.

11. American Diabetes Association. 2. Classification and diagnosis of diabetes: standards of medical care in diabetes-2020. Diabetes Care 2020;43(Suppl 1):S14-31.

12. Matthews DR, Hosker JP, Rudenski AS, Naylor BA, Treacher DF, Turner RC. Homeostasis model assessment: insulin resistance and beta-cell function from fasting plasma glucose and insulin concentrations in man. Diabetologia 1985;28:412-9.

13. Bergman RN, Ader M, Huecking K, Van Citters G. Accurate assessment of beta-cell function: the hyperbolic correction. Diabetes 2002;51 Suppl 1:S212-20.

14. Matsuda M, DeFronzo RA. Insulin sensitivity indices obtained from oral glucose tolerance testing: comparison with the eug- lycemic insulin clamp. Diabetes Care 1999;22:1462-70.

15. Chobanian AV, Bakris GL, Black HR, Cushman WC, Green LA, Izzo JL Jr, et al. The seventh report of the Joint National Committee on prevention, detection, evaluation, and treatment of high blood pressure: the JNC 7 report. JAMA 2003; 289:2560-72.

16. Klose G, Beil FU, Dieplinger H, von Eckardstein A, Foger B, Gouni-Berthold I, et al. New AHA and ACC guidelines on the treatment of blood cholesterol to reduce atherosclerotic cardiovascular risk. Wien Klin Wochenschr 2014;126:169-75.

17. Messerli FH, Hofstetter L, Rimoldi SF, Rexhaj E, Bangalore S. Risk factor variability and cardiovascular outcome: JACC review topic of the week. J Am Coll Cardiol 2019;73:2596-603.

18. Kim JA, Lee JS, Chung HS, Roh E, Lee YB, Hong SH, et al. Impact of visit-to-visit fasting plasma glucose variability on the development of type 2 diabetes: a nationwide populationbased cohort study. Diabetes Care 2018;41:2610-6.

19. Kodama S, Fujihara K, Ishiguro H, Horikawa C, Ohara N, Yachi Y, et al. Unstable bodyweight and incident type 2 diabetes mellitus: a meta-analysis. J Diabetes Investig 2017;8:501-9.

20. Rhee EJ, Han K, Ko SH, Ko KS, Lee WY. Increased risk for diabetes development in subjects with large variation in total cholesterol levels in 2,827,950 Koreans: a nationwide populationbased study. PLoS One 2017;12:e0176615.

21. Lee SH, Kim HS, Park YM, Kwon HS, Yoon KH, Han K, et al. HDL-cholesterol, its variability, and the risk of diabetes: a nationwide population-based study. J Clin Endocrinol Metab 2019;104:5633-41.

22. Cho EJ, Han K, Lee SP, Shin DW, Yu SJ. Liver enzyme variability and risk of heart disease and mortality: a nationwide population-based study. Liver Int 2020;40:1292-302.

23. Cho NH, Jang HC, Choi SH, Kim HR, Lee HK, Chan JC, et al. Abnormal liver function test predicts type 2 diabetes: a community-based prospective study. Diabetes Care 2007;30:25668.

24. Cho EJ, Kim SE, Suk KT, An J, Jeong SW, Chung WJ, et al. Current status and strategies for hepatitis B control in Korea. Clin Mol Hepatol 2017;23:205-11.

25. Kim BK, Jang ES, Kim JH, Park SY, Ahn SV, Kim HJ, et al. Current status of and strategies for hepatitis $\mathrm{C}$ control in South Korea. Clin Mol Hepatol 2017;23:212-8.

26. Miyake K, Miyake N, Kondo S, Tabe Y, Ohsaka A, Miida T. Seasonal variation in liver function tests: a time-series analysis of outpatient data. Ann Clin Biochem 2009;46(Pt 5):377-84. 